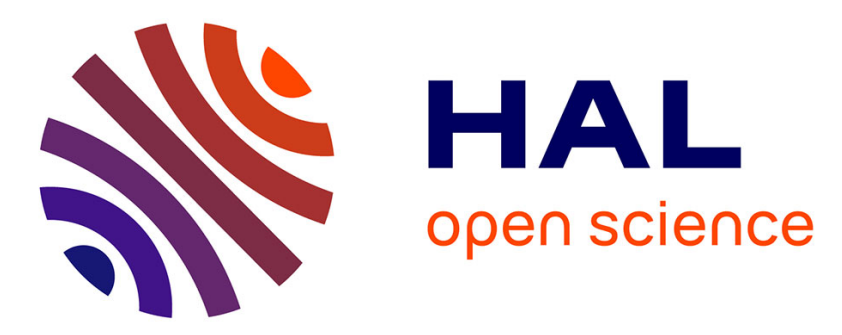

\title{
Near Infrared tunable source delivering ultra-short pulses based on an all normal dispersion fiber and a zero dispersion line
}

\author{
Coralie Fourcade-Dutin, Damien Bigourd
}

\section{- To cite this version:}

Coralie Fourcade-Dutin, Damien Bigourd. Near Infrared tunable source delivering ultra-short pulses based on an all normal dispersion fiber and a zero dispersion line. Applied Physics B Photophysics and Laser Chemistry, 2018, 124 (8), pp.154. 10.1007/s00340-018-7022-x . hal-03149325

\section{HAL Id: hal-03149325 \\ https://hal.science/hal-03149325}

Submitted on 22 Feb 2021

HAL is a multi-disciplinary open access archive for the deposit and dissemination of scientific research documents, whether they are published or not. The documents may come from teaching and research institutions in France or abroad, or from public or private research centers.
L'archive ouverte pluridisciplinaire HAL, est destinée au dépôt et à la diffusion de documents scientifiques de niveau recherche, publiés ou non, émanant des établissements d'enseignement et de recherche français ou étrangers, des laboratoires publics ou privés. 


\title{
Near Infrared tunable source delivering ultra-short pulses based on an all normal dispersion fiber and a zero dispersion line
}

\author{
Coralie Fourcade-Dutin ${ }^{\star}$, Damien Bigourd \\ Institut FEMTO-ST, Département Optique, UMR 6174, Université Bourgogne Franche-Comté-CNRS, 25030 Besançon, France
}

Received: 24/04/2018, Revised version: 21/06/2018

\begin{abstract}
A tunable laser source (820-1200 nm) delivering ultra-short pulses in the range of $40-100 \mathrm{fs}$ is investigated. It is based on the filtering of a continuum in the Fourier plane of a zero dispersion line without any phase compensator. The numerical simulations show the origin and the impact of the nonlinear chirp to guarantee ultra-short pulses.
\end{abstract}

\section{Introduction}

The generation of near infrared tunable (900-1300 nm) ultra-short pulses from a unique laser source at fixed center wavelength is highly important for many applications including non linear microscopy [1], optical coherence tomography [2] or laser pulse synchronization $[3,4]$. For example, optical parametric amplifier (OPA) requires at least two spectrally shifted pulses to seed and pump a non linear crystal $[5,6]$ or a fiber [7]. The synchronization of the pulses can be achieved passively when one pulse generates the other one through nonlinear processes enabling timing-jitter-free laser system with no complex electronic feedback loop [8]. When the OPA is driven by a high power laser, this optical synchronization is often achieved when a part of the beam (or its harmonics) pumps a nonlinear crystal or a bulk plate to generate a continuum [9] or super-fluorescence [10]. However, these methods require a high energy pulse in the range of few $\mu \mathrm{J}$. At lower energy, optical parametric oscillator with crystals [11] or fibers [12] can also be a good alternative at the expense of complexity and cost. In this case, very large tunability can be achieved, for example from 1400 to $1900 \mathrm{~nm}$. The tunable ultra-short pulse can also be obtained by nonlinear processes in photonic crystal fibers (PCF) as self-phase modulation SPM [13,14], soliton self-frequency shift $[3,4]$ or dispersive wave generation [15]. However, the dispersion feature of the PCF relatively to the experimental parameters defines the dynamics of the generated continuum [16]. Pumping the PCF in the anomalous dispersion regime near a single zero dispersion wavelength (ZDW) leads to soliton dynamics

\footnotetext{
* c.fourcadedutin@femto-st.fr
}

which are sensitive to pump fluctuation. This induces amplitude and timing jitter of the pulse and the temporal coherence properties are also degraded [17]. Alternatively, all normal dispersion fibers (ANDI) can be exploited to generate very large bandwidth [18] with smooth spectral intensity and phase. In this case, the continuum is considered as highly coherent since the nonlinear process is dominated by SPM and optical wave breaking (OWB) when the pump pulse duration is lower than few hundred of femtosecond $[19,20]$. Hence, this fiber is used to generate few cycle pulses when the spectral phase is compensated by chirped mirrors [21] or a pulse shaper device [22]. In this manuscript, we focus the investigation on the selection of tunable near infrared sub-100 fs pulses by filtering the continuum. Liu et al. [23] have used another approach and created a source delivering ultra-short pulses with a PCF owing two ZDWs. The tunability from 825 to $1210 \mathrm{~nm}$ is achieved with several optical bandpass filters and the power is adjusted to select the leftmost and rightmost lobes where the intensity is the highest. In this case, the pulse duration is higher than $100 \mathrm{fs}$ and is defined by the widths of the lobes and the filters. In our case, the total power is fixed to its maximum and the filtering is done with only one zero dispersion line combined with an iris in a Fourier plane without phase compensator to guarantee a large, smooth and practical tunability. We also demonstrate that the pulse duration does not significantly change with the increase of the power and therefore, the peak power of the selected pulses should be higher.

\section{Numerical simulation}

Numerical simulations are firstly conducted by integrating the nonlinear Schrödinger equation along the propagation length describing the evolution of the slowly varying total electric field $\mathbf{A}(\mathrm{z}, \tau)$ in the ANDI fiber :

$$
\begin{aligned}
& \frac{\partial A}{\partial z}=\sum_{k=2} \frac{i^{k+1}}{k !} \beta_{k} \frac{\partial^{k} A}{\partial \tau^{k}}+i \gamma\left(1+\frac{i}{\omega_{0}} \frac{\partial}{\partial \tau}\right) . \\
& {\left[A(z, \tau) \times \int R\left(\tau^{\prime}\right)\left|A\left(z, \tau-\tau^{\prime}\right)\right|^{2} d \tau^{\prime}\right]}
\end{aligned}
$$

The equation is solved with the standard split-step Fourier method. We assume a lossless fiber with a nonlinear coefficient equals to 
$\gamma=\mathbf{0 . 0 3 5} W^{-1} \mathrm{~km}^{-1} \cdot \omega_{0}$ is the central angular frequency of the input pulse. $\beta_{k}$ accounts for the k-th order fiber group-velocity dispersion. The typical dispersion curve is provided by the supplier (NKT Photonics) and is normal over a large spectral range (from $600 \mathrm{~nm}$ to $1300 \mathrm{~nm}$ ) with a maximum value of $-11.20 \mathrm{ps} /(\mathrm{km} . \mathrm{nm})$ at $1020 \mathrm{~nm}$. The nonlinear response includes both the instantaneous (Kerr effect) and delayed (Raman effect) contributions such as $R(\tau)=(1-$ $\left.f_{r}\right) \delta(\tau)+f_{r} h_{r}(\tau)\left(f_{r}=\mathbf{0 . 1 8}\right.$ and $h_{r}(\tau)$ is the delayed Raman response [24]). The experimental conditions are also used in the simulation : The pulse has a duration of $80 \mathrm{fs}$ at full width half maximum (FWHM) and a center wavelength of $1030 \mathrm{~nm}$. The repetition rate is 76 $\mathrm{MHz}$. Fig. 1.a displays the generated continuum in the fiber for $\mathrm{L}=3.5 \mathrm{~cm}$ and $\mathrm{L}=7 \mathrm{~cm}$ and for a power $\mathrm{P}=410$ $\mathrm{mW}$ and $\mathrm{P}=205 \mathrm{~mW}$.
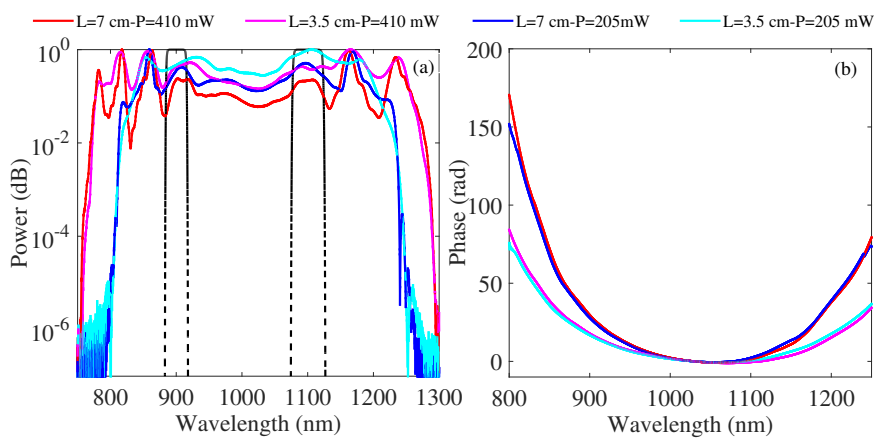

Fig. 1 (a) Spectrum and (b) phase of the simulated continuum generated in the ANDI fiber for several configurations. The shape of the filters are shown in dashed lines.

The spectrum broadens mainly due to SPM with some minor contributions of the OWB and self steepening [18]. The continuum extends from 750 to $1300 \mathrm{~nm}$ at maximum power. The nonlinearity combined with the dispersion leads to a nonlinear phase (Fig. 1.b) which is very important to predict the generation of ultrashort pulses. When L increases from $3.5 \mathrm{~cm}$ to $7 \mathrm{~cm}$, the bandwidth changes marginally while the spectral structures and phases are more pronounced. In order to check the possibility to generate ultra-short pulses by selecting parts of the full spectrum, the continuum is narrowed by bandpass filters centered at $\lambda_{c}=900$ and $\lambda_{c}=1100 \mathrm{~nm}$ with a bandwidth equals to $5.65 \mathrm{THz}$ at FWHM which correspond to $15 \mathrm{~nm}$ and $23 \mathrm{~nm}$, respectively (dashed lines-Fig. 1). The pulse duration is measured as a function of the input power. The output power of the filtered continuum increases with the input power (Fig. 2.a-b) while the pulse duration is approximatively constant around $80-85$ fs for $\mathrm{L}=3.5 \mathrm{~cm}$ near the Fourier transform (FT) limit (Fig. 2.c-d). The slight variation of the pulse duration with the power is due to the modification of the continuum structure. The pulse duration is approximatively the same at maximum power. Therefore, it is important to select the pulse at the maximum power to get the highest peak power. For the longer fiber, the pulse duration increases to 85-90 fs but is also relatively constant with the input power. The comparison with the FT limited pulse duration indicates that the chirp is more pronounced for the longer fiber. It is induced by SPM assisted by the dispersion.
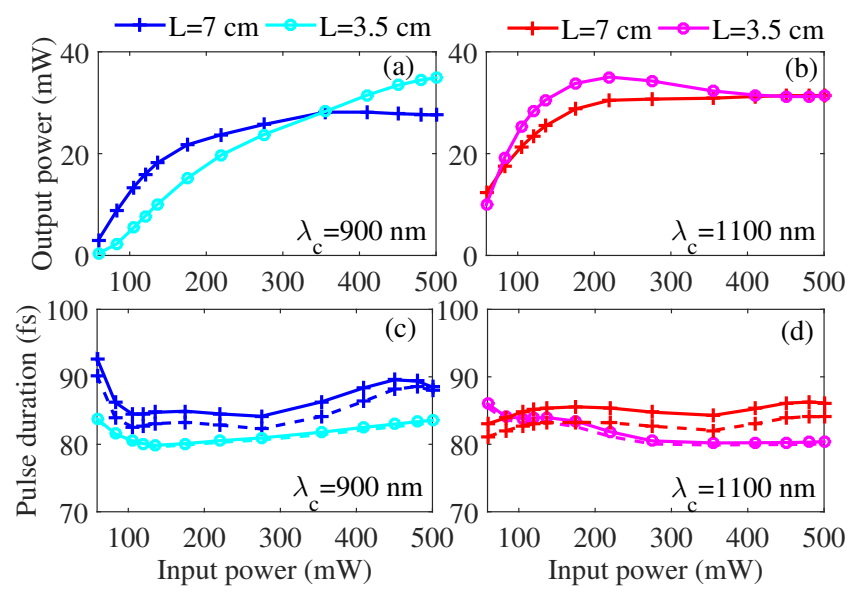

Fig. 2 Output power (a-b) and pulse duration (c-d) of the filtered continuum as a function of the input power. The dashed lines correspond to the duration of the FT limited pulse.

To reach shorter pulses, we investigate the impact of the filter bandwidth (at FWHM) on the duration (Fig. 3). As expected, the FT limited pulse duration decreases with a larger spectrum (dahed line-Fig. 3). However, the contribution of the spectral phase becomes more pronounced and therefore the pulse duration increases from its limits (solid line-Fig. 3). From a bandwidth equals to $\sim 28 \mathrm{~nm}$ at $900 \mathrm{~nm}$, the pulse duration increases suddenly for $\mathrm{L}=7 \mathrm{~cm}$ due to the local variation of spectral phase near the dip of the continuum (at $884 \mathrm{~nm}$ in Fig. 1.a). This impact of the phase can be avoided for shorter fiber (Fig. 3- cyan and magenta lines) since the whole spectral phase is decreased (Fig. 1.b). A similar but weaker effect is observed at $1100 \mathrm{~nm}$.

The tunability can be achieved by selecting different part of the continuum. The filter is tuned across the bandwidth. Its bandwidth equals to $5.65 \mathrm{THz}$ (e.g. 20 $\mathrm{nm}$ at $1030 \mathrm{~nm})$. Fig. 4 shows the pulse duration as a function of the center wavelength and some samples of the pulse shape are displayed when the fiber length equals to 3.5 or $7 \mathrm{~cm}$ (Fig. 4.b-c). For the short fiber, the 


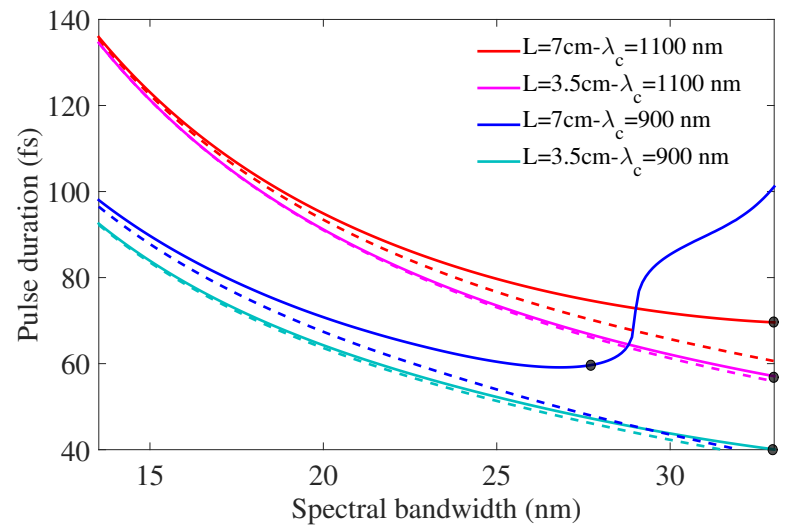

Fig. 3 Pulse duration as a function of the spectral bandwidth. $\mathrm{P}=410 \mathrm{~mW}$ in all cases. The dashed lines correspond to the duration of the FT limited pulses.
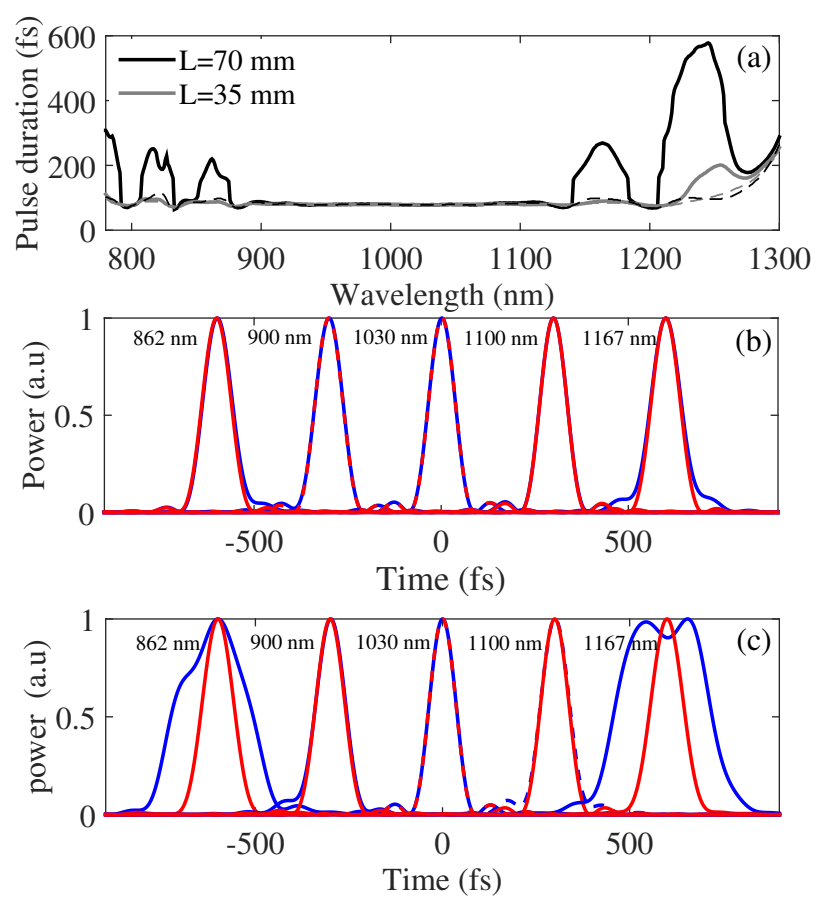

Fig. 4 (a) Pulse duration as a function of the center wavelength for a spectral bandwidth equals to $5.67 \mathrm{THz}$ (FWHM). The dashed lines correspond to the duration of the FT limited pulses. The pulse profiles are shown at various center wavelength for $\mathrm{L}=3.5 \mathrm{~cm}$ (b) and $\mathrm{L}=7 \mathrm{~cm}$ (c). The blue lines represent the profile of the filtered pulses while the red lines correspond to the Fourier limited pulses.

pulse duration is close to the limit imposed by the FT. For the longer fiber, the pulse duration remains short in the center of the continuum. However, the pulse duration increases more drastically at the edge of the spectrum due to the spectral phase. The increase of the pulse duration are observed when the spectrum in centered near a dip in the continuum (at $862 \mathrm{~nm}$ and $1167 \mathrm{~nm}$ ) as it was already observed in Fig. 3. This pulse lengthening can be avoided for shorter fiber. For example, the pulse duration at $862 \mathrm{~nm}$ or at $1167 \mathrm{~nm}$ is near the FT limit for $\mathrm{L}=3.5 \mathrm{~cm}$ (Fig.4.b-c).

\section{Experimental implementation}

The experimental set-up is displayed in Fig. 5. The seed laser is a commercial oscillator (Flint, LightConversion) which delivers a train of pulses at $76 \mathrm{MHz}$ with a duration of 80 fs at FWHM centered at 1030 nm (grey solid line in Fig. 6.c and Fig. 6.a). The total average power is $1.5 \mathrm{~W}$. A part of the beam is selected and injected in the ANDI fiber with a lens $(\mathrm{f}=4.5 \mathrm{~mm})$. The power at the output of the fiber is $410 \mathrm{~mW}$ and the coupling efficient is $\sim 33 \%$. The length $\mathrm{L}=7 \mathrm{~cm}$, is chosen to get a large and smooth continuum. It is a good compromise between good results and ease of use. Indeed, we did not want to use a too short fiber because it is often cleaved during this experiment. Moreover, we do not aim to perform a careful comparison with the simulation that does not include the exact values of the parameters (e.g. power, dispersion profile).

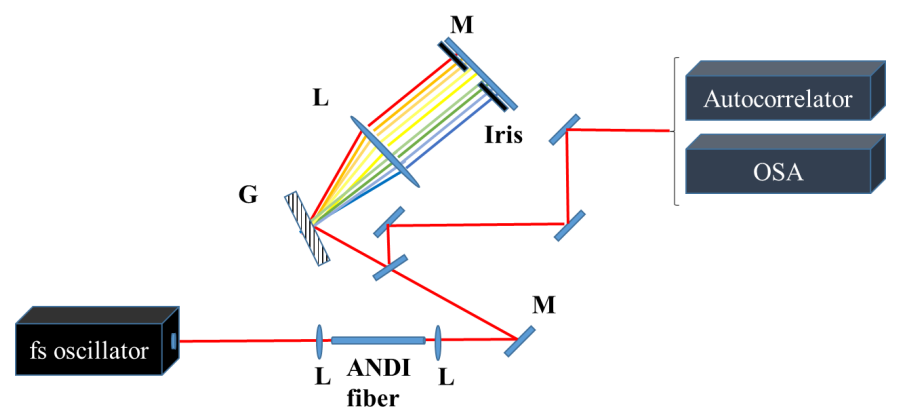

Fig. 5 Experimental set-up. M Mirror, L Lens, G Grating. The folded mirror placed on a translation stage. The grating is set on translation and rotation stages.

The continuum is measured with an optical spectrum analyzer (OSA) and the bandwidth extends from $760 \mathrm{~nm}$ to $1300 \mathrm{~nm}$. Firstly, we have performed a preliminary test in order to verify the possibility to obtain an ultrashort pulse at a selected wavelength. The continuum is narrowed by a low order super-Gaussian bandpass filter centered at $905 \mathrm{~nm}$ with a bandwidth equal to $25 \mathrm{~nm}$ at FWHM (Thorlabs- FL905-25) without any compressor. The filtered spectrum is shown in Fig. 6.a (black dashed line). The spectral modulation is weak within the bandwidth. The autocorrelation (AC) trace has a Gaussian shape with a pulse duration of 140 fs (Fig. 6.b-black solid line) while the FT limited duration of the AC is 80 fs (Fig. 6.b-black dashed line). Assuming a Gaussian profile (Fig. 6.b-green dotted line), we conclude that it is possible to generate a 100 fs pulse with some residual spectral phase when the spectrum is filtered in the continuum. In order to achieve a tunable filtering system with adjustable bandwidth, we develop a folded 4-f zero dispersion line composed of a 

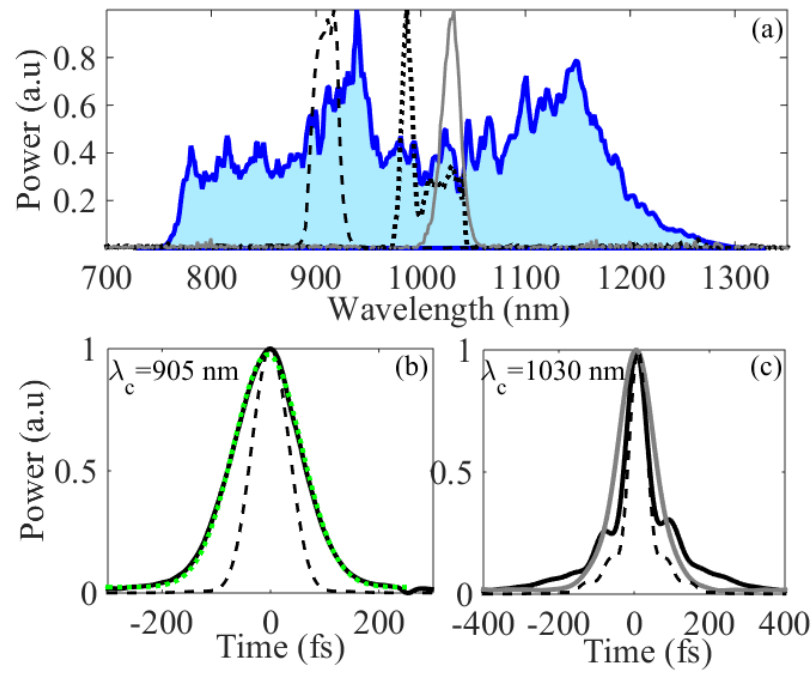

Fig. 6 (a) Continuum generated in a $7 \mathrm{~cm}$ long ANDI fiber for $\mathrm{P}=410 \mathrm{~mW}$ (blue line). Spectrum selected in the continuum by a bandpass filter at $905 \mathrm{~nm}$ (black dashed line) and at $\sim 1030 \mathrm{~nm}$ with the $4 \mathrm{f}$-line (black dotted line). Spectrum of the oscillator (grey solid line). (b) AC pulse shape (black solid line) and its fit with a Gaussian profile (green dotted line) when the spectrum is centered at $905 \mathrm{~nm}$. The black dashed line corresponds to the FT limited pulse. (c) AC pulse shapes of the filtered continuum centered at $1030 \mathrm{~nm}$ (black solid line) and the oscillator (gray solid line). The black dashed line corresponds to the FT limited AC.

grating with 1200 lines/mm (Thorlabs, GR50-1210), a lens $(\mathrm{f}=100 \mathrm{~mm})$ and a flat mirror (Fig. 5). The grating and the folded mirror are placed on translation stages to optimize the dispersion induced by the lens at each wavelength. Firstly, the line is aligned with the output of the oscillator without the fiber and we check that it does not add any dispersion by measuring the pulse duration. Then, the continuum is injected in the 4f-line and an iris is placed in the Fourier plane to select both the central wavelength and the spectral bandwidth. The spectrum and the $\mathbf{A C}$ duration are measured with an OSA and a non collinear autocorrelator, respectively. If not stated otherwise, the durations given in the following correspond to the autocorrelation trace width. The optimal bandwidth is chosen such as the pulse duration is the lowest for a minimum spectral bandwidth. According to the simulation shown in Fig. 3 (black circles), the shortest pulse duration is optimum for a well-defined bandwidth.

The AC duration is firstly measured when the spectrum is narrowed around $1030 \mathrm{~nm}$ with an iris placed in the Fourier plane (black dotted line, Fig. 6.b). The autocorrelation pulse duration is 66 fs (FWHM) (black solid line, Fig. 6.c). The FT limited duration of the AC trace is 56 fs (FWHM). It confirms that the pulse has a residual spectral phase which is not compensated in the experiment. The tunability is achieved by injecting the
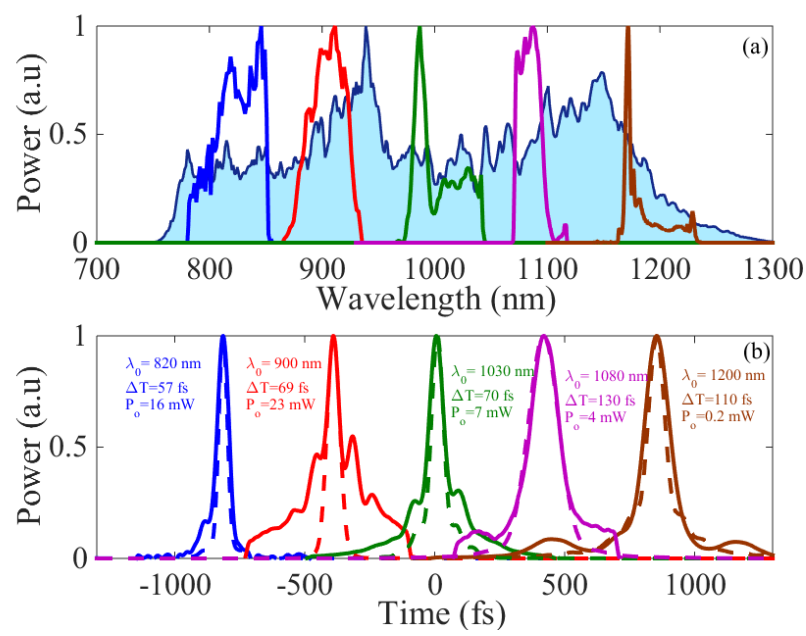

Fig. 7 (a) Selection of filtered spectrum in the 4-f line. (b) Corresponding AC traces.

same continuum in the 4 -f zero dispersion line. For each spectral selection, the pulse duration is measured with the autocorrelator. For a short scan of the wavelength, the iris can be translated in front of the folded mirror. However, as the continuum has a very large bandwidth, the spectrum is clipped on the side of the mirror and the lens. Therefore, the grating is rotated for the long scan of the spectrum, and the fine tuning is achieved with the iris. In each case, the bandwidth is chosen to get the shortest duration for the minimal spectral bandwidth. The distances between the grating, the lens and the folded mirrors are optimized to limit both the spectral phase added from the 4 -f line and the spatial chirp. The alignment procedure takes only few minutes and it can be simplified with a reflective focusing optic instead of the lens. Several examples are shown in Fig. 7. The structures in the filtered spectrum are mainly due to the continuum and to the spectral transmission of the 4 -f line. The pulse durations of the autocorrelation trace range from 57 fs to 130 fs when the central wavelength varies from $820 \mathrm{~nm}$ to $1200 \mathrm{~nm}$. The AC do not change significantly during few hours and we evaluate the stability at 5-10 \%. The pedestal observed in Fig. 7.b are mainly due to the spectral phase and not to the modulated spectrum since the FT limited durations of the AC have bell-shaped profiles. We observe that the pedestals are more pronounced at the lower wavelength part. This spectral phase can be compensated with several systems as a pulse shaper [22] or chirped mirrors [21] but they need to be specifically optimized for a relatively narrow bandwidth. The weak output power from $0.2 \mathrm{~mW}$ to $23 \mathrm{~mW}$ (Fig. 7) is due to both the filtering and the spectral function of the grating. However, this level is sufficient to seed a fiber based optical parametric amplifier [25]. 


\section{Conclusion}

We have presented a practical and cost-effective method enabling the realization of a near infrared source delivering pulses with a duration of 40-100 fs. Numerical simulations demonstrate the possibilty to deliver ultra-short pulses by filtering a part of the spectrum even at the maximum input power. The tunability is experimentally achieved from $800 \mathrm{~nm}$ to $1200 \mathrm{~nm}$ by selecting a part of a continuum with a zero dispersion line. The shortest pulse duration (40 fs assuming a Gaussian shape) is obtained at $820 \mathrm{~nm}$ without any phase compensator. We have also showed that the uncompensated spectral phase may deteriorate the pulse duration but the impact depends strongly on the experimental condition as the fiber length and the selected center wavelength. In a future work, the pulses will be amplified in a fiber through an optical parametric process.

Acknowledgements This work has been supported by the Agence Nationale de la Recherche (Labex Action ANR-11-LABX-0001-01 and FiberAmp projects ANR16-CE24-0009) and the conseil Régional de FrancheComté.

\section{References}

1. S. R. Domingue, R. A. Bartels, Optics Express 22, 30777 (2014)

2. K. Sumimura,Y. Genda, T. Ohta,K. Itoh, N. Nishizawa, Optics Letters 35, 3631 (2010)

3. J. Rothhardt, A. M. Heidt, S. Hdrich, S. Demmler, J. Limpert, A. Tunermann, J. Opt. Soc. Am. B 29, 1257 (2012)

4. K. Mecseki, D. Bigourd, S. Patankar, N. H. Stuart,R. A. Smith, Applied Optics 53, 2229 (2014)

5. S. A. Rezvani, Z. Hong, X. Pang, S. Wu, Q. Zhang, P. Lu, Optics Letter 42, 3367 (2017)

6. D. Bigourd, S. Patankar, S. I. Olsson Robbie, H. W. Doyle, K. Mecseki, N. Stuart, K. Hadjicosti, N. Leblanc, G. H. C. New, R. A. Smith, Applied Physics B 113, 627 (2013)

7. D. Bigourd, L. Lago, A. Mussot, A. Kudlinski, J.F. Gleyze, E. Hugonnot, Optics Letters 42, 3480 (2010)

8. C. Y. Teisset, N. Ishii, T. Fuji, T. Metzger, S. Kohler, R. Holzwarth, A. Baltuska, A. M. Zheltikov, F. Krausz, Optics Express 13, 6550 (2005)

9. N. Garejev, G. Tamosauskas, A. Dubietis, J. Opt. Soc. Am. B 34, 88 (2017)

10. G. P. Banfi and P. Di Trapani, R. Danielius, A. Piskarskast, R. Righini, I. Santa, Optics Letters 18, 1547 (1993)

11. A. Esteban-Martin,V. Ramaiah-Badarla, V. Petrov, M. Ebrahim-Zadeh Optics Letters 36, 1671 (2011)

12. T.Gottschall, T. Meyer, M. Schmitt, J. Popp, J. Limpert, A. Tunnermann Optics Express 23, 23968 (2015)

13. H. Y. Chung, W. Liu, Q. Cao, L. Song, F. X. Kartner, G. Chnag Optics Express 26, 3684 (2018)
14. B. Metzger, A. Steinmann, H. Giessen, Optics Express 19, 24354 (2011)

15. J. C. Travers, J. R. Taylor, Optics Letters 34, 115 (2009)

16. J. M. Dudley, G. Genty, S. Coen, Rev. Mod. Phys. 78, 1135 (2006)

17. M. Klimczak, G. Sobon, R. Kasztelanic, K. M. Abramski, R.Buczynski, Scientific Reports 6:19284, 1 (2016)

18. A. M. Heidt, J. Opt. Soc. Am. B 27, 550 (2010)

19. C. Finot, B. Kibler, L. Provost, S. Wabnitz, J. Opt. Soc. Am. B 25, 1938 (2008)

20. A. M. Heidt, J. S. Feehan, J. H. V. Price, T. Feurer, J. Opt. Soc. Am. B 34, 764 (2017)

21. A. M. Heidt, J. Rothhardt, A. Hartung, H. Bartelt, E. G. Rohwer, J. Limpert, A. Tunnermann, Optics Express, 19, $13873(2011)$

22. S. Demmler, J. Rothhardt, A. M. Heidt, A. Hartung, E. G. Rohwer, H. Bartelt, J. Limpert, A. Tunnermann, Optics Express, 19, 20151 (2011)

23. W. Liu,C. Li, Z. Zhnag, F. X. Kartner, G. Chang, Optics Express 24, 15328 (2016)

24. A. S. Y. Hsieh, G. K. L. Wong, S. G. Murdoch, S. Coen, F. Vanholsbeeck, R. Leonhardt, J. D. Harvey, Optics Express 15, 8104 (2007)

25. D. Bigourd, P. Beaure dAugers, J. Dubertrand, E. Hugonnot, A. Mussot, Optics Letters 39, 3782 (2014) 